Eng. Appl. Sci. Lett., Vol. 1(2018), No. 2, pp. 10 - 22

Website: https://pisrt.org/psr-press/journals/easl/

ISSN: 2617-9709 (Online) 2617-9695 (Print)

http://dx.doi.org/10.30538/psrp-easl2018.0007

\title{
BOUNDEDNESS OF LITTLEWOOD-PALEY OPERATORS WITH VARIABLE KERNEL ON WEIGHTED HERZ SPACES WITH VARIABLE EXPONENT
}

\author{
AFIF ABDALMONEM ${ }^{1}$, OMER ABDALRHMAN, SHUANGPING TAO
}

\begin{abstract}
Let $\Omega \in L^{\infty}\left(\mathbb{R}^{n}\right) \times L^{2}\left(S^{n-1}\right)$ be a homogeneous function of degree zero. In this article, we obtain some boundedness of the parameterized Littlewood-Paley operators with variable kernels on weighted Herz spaces with variable exponent.

Index Terms: Parameterized Littlewood-Paley operators; variable kernel; weighted Herz spaces; Muckenhoupt; variable exponents.
\end{abstract}

\section{Introduction}

The boundedness of Littlewood-Paley operators on function spaces are one of the very important tools, not only in harmonic analysis, but also in potential theory and in partial differential equations (see[1, 2, 3, 4, 5, 6], for details). In 2004, Ding, Lin and Shao [7] investigated the $L^{2}$-boundedness for a class of Marcinkiewicz integral operators with variable kernels $\mu_{\Omega}$ and $\mu_{\Omega, s}$ related to the Littlewood-Paley function $\mu_{\Omega, \lambda}^{*}$ and the area integral $g_{\lambda}^{*}$. In 2006, the authors [8] proved the $L^{p}$-boundedness of the Littlewood-Paley operators with variable kernels. In 2009, Xue and Ding [9] established the weighted estimate for Littlewood-Paley operators and their commutators.

In 1960, Hörmander [10] introduced the parameterized Littlewood-Paley operators for the first time. Now, let us recall the definitions of the parameterized Lusin area integral and Littlewood-Paley $g_{\lambda}^{*}$ function.

Let $S^{n-1}(n \geq 2)$ be the unit sphere in $\mathbb{R}^{n}$ with normalized Lebesgue measure $\mathrm{d} \sigma\left(x^{\prime}\right)$. Take $\Omega(x, z) \in L^{\infty}\left(\mathbb{R}^{n}\right) \times L^{r}\left(S^{n-1}\right)(r \geq 1)$ to be a homogeneous function

Received 20-11-2018. Revised 16-12-2018.

1 Corresponding Author

(c) 2018 Afif Abdalmonem, Omer Abdalrhman, Shuangping Tao. This is an open access article distributed under the Creative Commons Attribution License, which permits unrestricted use, distribution, and reproduction in any medium, provided the original work is properly cited. 
of degree zero and

$$
\int_{s^{n-1}} \Omega\left(x, z^{\prime}\right) \mathrm{d} \sigma\left(z^{\prime}\right)=0, \text { for all } x \in \mathbb{R}^{n},
$$

where $\Omega$ satisfies the following conditions:

(1) For any $x, z \in \mathbb{R}^{n}$ and any $\lambda>0$, we have $\Omega(x, \lambda z)=\Omega(x, z)$;

(2) $\|\Omega\|_{L^{\infty}\left(\mathbb{R}^{n}\right) \times L^{r}\left(S^{n-1}\right)}:=\sup _{r>0, y \in \mathbb{R}^{n}}\left(\int_{s^{n-1}}\left|\Omega\left(r z^{\prime}+y, z^{\prime}\right)\right|^{r} \mathrm{~d} \sigma\left(z^{\prime}\right)\right)^{\frac{1}{r}}<\infty$.

The parameterized Littlewood-Paley operators $\mu_{\Omega, s}^{\rho}$ and $\mu_{\Omega, \lambda}^{*, \rho}$ with variable kernels, which are related to the Lusin area integral and the Littlewood-Paley $g_{\lambda}^{*}$ function are defined by

$$
\mu_{\Omega, s}^{\rho}(f)(x)=\left(\iint_{\Gamma(x)}\left|\frac{1}{t^{\rho}} \int_{|y-z| \leq t} \frac{\Omega(y, y-z)}{|y-z|^{n-\rho}} f(z) \mathrm{d} z\right|^{2} \frac{\mathrm{d} y \mathrm{~d} t}{t^{n+1}}\right)^{\frac{1}{2}}
$$

and

$$
\begin{aligned}
& \mu_{\Omega, \lambda}^{*, \rho}(f)(x)= \\
& \left(\iint_{\mathbb{R}_{+}^{n+1}}\left(\frac{t}{t+|x-y|}\right)^{\lambda n}\left|\frac{1}{t^{\rho}} \int_{|y-z| \leq t} \frac{\Omega(y, y-z)}{|y-z|^{n-\rho}} f(z) \mathrm{d} z\right|^{2} \frac{\mathrm{d} y \mathrm{~d} t}{t^{n+1}}\right)^{\frac{1}{2}},
\end{aligned}
$$

where $\Gamma(x)=\left\{(y, t) \in \mathbb{R}_{+}^{n+1}:|x-y|<t\right.$ and $\left.\lambda>1\right\}$.

In 2013, Wei and Tao [11] investigated the boundedness of parameterized Littlewood Paley operators on weighted weak Hardy spaces. Lin and Xuan [12] established the boundedness for commutators of parameterized Littlewood-Paley operators and area integrals on weighted Lebesgue spaces $L^{p}(w)$.

The theory of the variable exponent function spaces has been rapidly developed after the work [13], where Kováćik and Rákosník have clarified fundamental properties of Lebesgue spaces with variable exponent. After that, many researchers have been interested in the theory of the variable exponent spaces (see $[14,15,16,17,18,19,20])$.

The generalization of the Muckenhoupt weights with variable exponent $A_{p(\cdot)}$ has been considered in [21, 22, 23, 24]. The equivalence between the Muckenhoupt condition and the boundedness of the Hardy-Littlewood maximal operator on weighted Lebesgue spaces with variable exponent were discussed in [21, 22]. After that, Cruz-Uribe and Wang [25] proved the boundedness of some classical operators on weighted Lebesgue spaces with variable exponent $L^{p(\cdot)}(w)$.

Recently, Izuki and Noi [26] introduced the weighted Herz spaces with variable exponent, and also studied the boundedness of fractional integrals on those spaces.

In this paper, we establish the boundedness of parameterized Littlewood-Paley operators with variable kernels on weighted Herz spaces with variable exponent 
$\dot{K}_{p(\cdot)}^{\alpha, q}(w)$. Let $E$ be a Lebesgue measurable set in $\mathbb{R}^{n}$ with measure $|E|>0, \chi_{E}$ means its characteristic function. We shall recall some definitions.

Definition 1.1. Let $p(\cdot): E \rightarrow[1, \infty)$ be a measurable function. The variable exponent Lebesgue space is defined as

$L^{p(\cdot)}(E)=\left\{f\right.$ is measurable $: \int_{E}\left(\frac{|f(x)|}{\eta}\right)^{p(x)} \mathrm{d} x<\infty$ for some constant $\left.\eta>0\right\}$.

The space $L_{\mathrm{loc}}^{p(\cdot)}(E)$ is defined as

$$
L_{\text {loc }}^{p(\cdot)}(E)=\left\{f \text { is measurable }: f \in L^{p(\cdot)}(K) \text { for all compact } K \subset E\right\} .
$$

The Lebesgue spaces $L^{p(\cdot)}(E)$ is a Banach spaces with the norm defined as

$$
\|f\|_{L^{p(\cdot)}(E)}=\inf \left\{\eta>0: \int_{E}\left(\frac{|f(x)|}{\eta}\right)^{p(x)} \mathrm{d} x \leq 1\right\} .
$$

We denote $p_{-}=\operatorname{ess} \inf \{p(x): x \in E\}, p_{+}=\operatorname{ess} \sup \{p(x): x \in E\}$, then $\mathcal{P}(E)$ consists of all $p(\cdot)$ satisfying $p_{-}>1$ and $p_{+}<\infty$.

Definition 1.2. [27] Let $p(\cdot): \mathbb{R}^{n} \rightarrow[1, \infty)$. A measurable function $p(\cdot)$ is said to be globally log-Höder continuous if it satisfies

(1) $|p(x)-p(y)| \leq \frac{1}{-\log (|x-y|)}, x, y \in \mathbb{R}^{n},|x-y| \leq 1 / 2$;

(2) $\left|p(x)-p_{\infty}\right| \leq \frac{1}{\log (e+|x|)}, x \in \mathbb{R}^{n}$,

for some $p_{\infty} \geq 1$. The set of $p(\cdot)$ satisfying conditions (1) and (2) is denoted by $L H\left(\mathbb{R}^{n}\right)$.

We know that, if $p(\cdot) \in L H\left(\mathbb{R}^{n}\right) \cap \mathcal{P}\left(\mathbb{R}^{n}\right)$, the Hardy-Littlewood maximal operator $M$ is bounded on $L^{p(\cdot)}\left(\mathbb{R}^{n}\right)$ (see[28]).

Definition 1.3. [29] Suppose that $p(\cdot) \in \mathcal{P}\left(\mathbb{R}^{n}\right)$ and $w$ is a weight function. The weighted Lebesgue spaces with variable exponent $L^{p(\cdot)}(w)$ is the set of all complex-valued measurable function $f$ such that $f w^{1 / p(\cdot)} \in L^{p(\cdot)}\left(\mathbb{R}^{n}\right)$. The space $L^{p(\cdot)}(w)$ is a Banach space equipped with the norm

$$
\|f\|_{L^{p(\cdot)}(w)}=\left\|f w^{1 / p(\cdot)}\right\|_{L^{p(\cdot)}} .
$$

$p^{\prime}(\cdot)$ is the conjugate of $p(\cdot)$ such that $\frac{1}{p^{\prime}(\cdot)}+\frac{1}{p(\cdot)}=1$. Next, we introduce the classical Muckenhoupt $A_{p}$ weight.

Definition 1.4. [30] Let $1<p<\infty$, then $w \in A_{p}$ for every cube $Q$,

$$
\left(\frac{1}{|Q|} \int_{Q} w(x) \mathrm{d} x\right)\left(\frac{1}{|Q|} \int_{Q} w(x)^{1-p^{\prime}} \mathrm{d} x\right)^{p-1} \leq C<\infty .
$$

We say that $w \in A_{1}$ if it satisfies $M w(x) \leq w(x)$ for all $x \in \mathbb{R}^{n}$. The set $A_{1}$ consists of all Muckenhoupt $A_{1}$ weights. 
Definition 1.5. $[21,25]$ Given $p(\cdot) \in \mathcal{P}\left(\mathbb{R}^{n}\right)$ and a weight $w$, then $w \in A_{p(\cdot)}$ if

$$
\sup _{\text {B:ball }}|B|^{-1}\left\|w^{1 / p(\cdot)} \chi_{B}\right\|_{L^{p(\cdot)}(w)}\left\|w^{-1 / p(\cdot)} \chi_{B}\right\|_{L^{p^{\prime}(\cdot)}(w)}<\infty .
$$

Definition 1.6. [25] Given $p_{1}(\cdot), p_{2}(\cdot) \in \mathcal{P}\left(\mathbb{R}^{n}\right)$ and $1 / p_{1}(x)-1 / p_{2}(x)=\mu / n$ such that $0<\mu<n$. Then $w \in A_{p_{1}(\cdot), p_{2}(\cdot)}$ if

$$
\left\|w \chi_{B}\right\|_{L^{p_{2}(\cdot)}}\left\|w^{-1} \chi_{B}\right\|_{L^{p_{1}^{\prime}(\cdot)}} \leq|B|^{\frac{n-\mu}{n}}
$$

holds for all balls $B \in \mathbb{R}^{n}$.

Definition 1.7. [25] Let $p(\cdot) \in \mathcal{P}\left(\mathbb{R}^{n}\right)$ and $w$ be a weight. We say that $(p(\cdot), w)$ is an $M$-pair if the maximal operator $M$ is bounded on $L^{p(\cdot)}(w)$ and $L^{p^{\prime}(\cdot)}\left(w^{-1}\right)$.

Now, we to need to give the definition of weighted Herz space with variable exponent. For all $k \in \mathbb{Z}$, we denote $B_{k}=\left\{x \in \mathbb{R}^{n}:|x| \leq 2^{k}\right\}, C_{k}=B_{k} \backslash B_{k-1}$, $\chi_{k}=\chi_{C_{k}}$.

Definition 1.8. [26] Suppose that $p(\cdot) \in \mathcal{P}\left(\mathbb{R}^{n}\right), 0<q<\infty, \alpha \in \mathbb{R}$. The homogeneous weighted Herz space with variable exponent $\dot{K}_{p(\cdot)}^{\alpha, q}(w)$ is the collection of $f \in L_{\mathrm{loc}}^{p(\cdot)}\left(\mathbb{R}^{n} \backslash\{0\}, w\right)$ such that

$$
\|f\|_{\dot{K}_{p(\cdot)}^{\alpha, q}(w)}:=\left(\sum_{k=-\infty}^{\infty} 2^{\alpha q k}\left\|f \chi_{k}\right\|_{L^{p(\cdot)}(w)}^{q}\right)^{1 / q}<\infty .
$$

It is easy to see that if $w=1$, then $\dot{K}_{p(\cdot)}^{\alpha, q}(w)=\dot{K}_{p(\cdot)}^{\alpha, q}\left(\mathbb{R}^{n}\right)$ is the Herz space with variable exponen [17]. If $w=1$ and $p(\cdot)=p$, then $\dot{K}_{p(\cdot)}^{\alpha, q}(w)=\dot{K}_{p}^{\alpha, q}\left(\mathbb{R}^{n}\right)$ is the classical Herz space introduced in [31]. If $p(\cdot)=p$, then $\dot{K}_{p(\cdot)}^{\alpha, q}(w)=\dot{K}_{p}^{\alpha, q}(w)$ is the weighted Herz space [32].

Definition 1.9. We say a kernel function $\Omega(x, z)$ satisfies the $L^{r}$-Dini condition $(r \geq 1)$, if

$$
\int_{0}^{1} \frac{\omega_{r}(\delta)}{\delta}\left(1+|\log \delta|^{\sigma}\right) \mathrm{d} \delta<\infty
$$

where $\omega_{r}(\delta)$ denotes the integral modulus of continuity of order $r$ of $\Omega$ defined by

$$
\omega_{r}(\delta)=\sup _{x \in \mathbb{R}^{n},|\rho|<\delta}\left(\int_{\delta^{n-1}}\left|\Omega\left(x, \rho z^{\prime}\right)-\Omega\left(x, z^{\prime}\right)\right|^{r} \mathrm{~d} \sigma\left(z^{\prime}\right)\right)^{\frac{1}{r}},
$$

where $\rho$ is the rotation in $\mathbb{R}^{n},\|\rho\|=\sup _{z^{\prime} \in S^{n-1}}\left\|\rho z^{\prime}-z^{\prime}\right\|$. 


\section{Preliminaries and notations}

In order to prove our main theorems, we need the following Lemmas.

Lemma 2.1. [3] Suppose that $X \subset \mathcal{M}$ is a Banach function space.

(1) (The generalized Hölder inequality) For all $f \in X$ and $g \in X^{\prime}$, we have

$$
\int_{\mathbb{R}^{n}}|f(x) g(x)| \mathrm{d} x \leq\|f\|_{X}\|g\|_{X} .
$$

(2) For all $f \in X$, we have

$$
\sup \left\{\left|\int_{\mathbb{R}^{n}} f(x) g(x) \mathrm{d} x\right|:\|g\|_{X^{\prime}} \leq 1\right\}=\|f\|_{X} .
$$

In particular, space $\left(X^{\prime}\right)^{\prime}=X$.

As an application of the generalized Hölder inequality above, we have the following Lemma.

Lemma 2.2. Let $X$ be a Banach function space, we have

$$
1 \leq \frac{1}{|B|}\left\|\chi_{B}\right\|_{X}\left\|\chi_{B}\right\|_{X^{\prime}}
$$

hold for all balls $B$.

Lemma 2.3. [24] Let $X$ be a Banach function space. If the Hardy-Littlewood maximal operator $M$ is weakly bounded on $X$, that is

$$
\left\|\chi_{\{M f>\lambda\}}\right\|_{X} \leq \lambda^{-1}\|f\|_{X},
$$

holds for all $f \in X$ and $\lambda>0$ l, then we get

$$
\sup _{\text {B:Ball }} \frac{1}{|B|}\left\|\chi_{B}\right\|_{X}\left\|\chi_{B}\right\|_{X^{\prime}}<\infty .
$$

Remark 2.1. [29] The weighted Banach function space $X\left(\mathbb{R}^{n}, W\right)$ is a Banach function space equipped the norm $\|f\|_{X\left(\mathbb{R}^{n}, W\right)}:=\|f W\|_{X}$. The associated space of $X\left(\mathbb{R}^{n}, W\right)$ is a Banach function space and equals $X^{\prime}\left(\mathbb{R}^{n}, W^{-1}\right)$.

Remark 2.2. If $p_{1}(\cdot) \in \mathcal{P}\left(\mathbb{R}^{n}\right)$, by comparing the definition of the weighted Banach function space with weighted variable Lebesgue space, we have

(1) If $X=L^{p_{1}(\cdot)}\left(\mathbb{R}^{n}\right)$ and $W=w$, then we obtain

$$
L^{p_{1}(\cdot)}\left(\mathbb{R}^{n}, w\right)=L^{p_{1}(\cdot)}\left(w^{p_{1}(\cdot)}\right) .
$$

(2) If $X=L^{p_{1}^{\prime}(\cdot)}\left(\mathbb{R}^{n}\right)$ and $W=w^{-1}$. Using Lemma 2.3, we obtain

$$
L^{p_{1}^{\prime}(\cdot)}\left(\mathbb{R}^{n}, w^{-1}\right)=L^{p_{1}^{\prime}(\cdot)}\left(w^{-p_{1}^{\prime}(\cdot)}\right)=\left(L^{p_{1}(\cdot)}\left(w^{p_{1}(\cdot)}\right)\right)^{\prime} .
$$


Lemma 2.4. [33] Suppose that $X$ is a Banach space. Let $M$ be bounded on the associated space $X^{\prime}$. Then there exists a constant $0<\delta<1$ such that

$$
\frac{\left\|\chi_{E}\right\|_{X}}{\left\|\chi_{B}\right\|_{X}} \leq\left(\frac{|E|}{|B|}\right)^{\delta}
$$

holds for all balls $B$ and all measurable sets $E \subset B$.

Lemma 2.5. [26] Suppose that $p_{1}(\cdot) \in L H\left(\mathbb{R}^{n}\right) \cap \mathcal{P}\left(\mathbb{R}^{n}\right)$ and $w^{p_{1}(\cdot)} \in A_{1}$. Let $M$ be a bounded on $L^{p_{1}(\cdot)}\left(w^{p_{1}(\cdot)}\right)$ and $L^{p_{1}^{\prime}(\cdot)}\left(w^{-p_{1}^{\prime}(\cdot)}\right)$, then there exist constants $\delta_{1}, \delta_{2} \in(0,1)$ such that

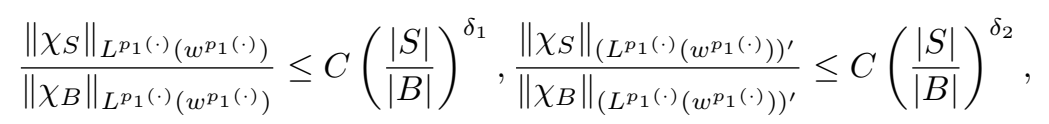

hold for all balls $B$ and all measurable sets $S \subset B$.

Lemma 2.6. [34] Suppose that $\Omega \in L^{\infty}\left(\mathbb{R}^{n}\right) \times L^{2}\left(S^{n-1}\right)$ satisfies equation (1) and definition (1.9), $\lambda>2,2 \rho-n>0,1<p<\infty$. Then for all $f \in L^{q}(w)$ there exists $C>0$ independent of $f$ such that

$$
\left\|\mu_{\Omega, s}^{\rho} f\right\|_{L^{q}(w)} \leq C\|f\|_{L^{q}(w)}
$$

and

$$
\left\|\mu_{\Omega, \lambda}^{*, \rho} f\right\|_{L^{q}(w)} \leq C\|f\|_{L^{q}(w)} .
$$

Lemma 2.7. [25] Assume that for $p_{0}, 1<p_{0}<\infty$ and every $w_{0} \in A_{p_{0}}$,

$$
\int_{\mathbb{R}^{n}} f(x)^{p_{0}} w_{0}(x) \mathrm{d} x \leq \int_{\mathbb{R}^{n}} g(x)^{p_{0}} w_{0}(x) \mathrm{d} x, \quad(f, g) \in \mathcal{F} .
$$

Then for any $M$-pair $(p(\cdot), w)$,

$$
\|f\|_{L^{p(\cdot)}(w)} \leq C\|g\|_{L^{p(\cdot)}(w)}, \quad(f, g) \in \mathcal{F}
$$

Lemma 2.7 holds for $p_{0}=1$ and the maximal operator is bounded on $L^{p^{\prime}(\cdot)}\left(w^{-1}\right)$. We know the Hardy-Littlewood maximal operator is bounded on $L^{p(\cdot)}(w)$ and $L^{p^{\prime}(\cdot)}\left(w^{-p^{\prime}(\cdot)}\right)$ (see [33]).

Combining Lemma 2.6 with Lemma 2.7, we obtain the following conclusion.

Corollary 2.8. Let $p(\cdot) \in L H\left(\mathbb{R}^{n}\right) \cap \mathcal{P}\left(\mathbb{R}^{n}\right), \Omega \in L^{\infty}\left(\mathbb{R}^{n}\right) \times L^{r}\left(S^{n-1}\right)(r \geq 1)$ and $w \in A_{p(\cdot)}$. Then the parameterized Littlewood-Paley operators $\mu_{\Omega, s}^{\rho}$ and $\mu_{\Omega, \lambda}^{*, \rho}$ with variable kernels are bounded on $L^{p(\cdot)}(w)$.

\section{Main Theorems and their proofs}

In this section, we will prove the boundedness of the parameterized LittlewoodPaley operators with variable kernels on variable weighted Herz spaces. 
Theorem 3.1. Let $p(\cdot) \in L H\left(\mathbb{R}^{n}\right) \cap \mathcal{P}\left(\mathbb{R}^{n}\right), 0<q_{1} \leq q_{2}<\infty, \lambda>2,2 \rho-n>0$ and $\Omega \in L^{\infty}\left(\mathbb{R}^{n}\right) \times L^{2}\left(S^{n-1}\right)$ satisfies (1.1) and (1.2). If $w^{p(\cdot)} \in A_{1}$ and $-n \delta_{1}<$ $\alpha<n \delta_{2}$, where $\delta_{1}, \delta_{2}$ are the constants in Lemma 2.5, then the operator $\mu_{\Omega, s}$ is bounded from $\dot{K}_{p_{1}(\cdot)}^{\alpha, q_{2}}\left(w^{p_{1}(\cdot)}\right)$ to $\dot{K}_{p_{1}(\cdot)}^{\alpha, q_{1}}\left(w^{p_{1}(\cdot)}\right)$.

Theorem 3.2. Let $p(\cdot) \in L H\left(\mathbb{R}^{n}\right) \cap \mathcal{P}\left(\mathbb{R}^{n}\right), 0<q_{1} \leq q_{2}<\infty, \lambda>2,2 \rho-n>0$ and $\Omega \in L^{\infty}\left(\mathbb{R}^{n}\right) \times L^{2}\left(S^{n-1}\right)$ satisfies (1.1) and (1.2). If $w^{p(\cdot)} \in A_{1}$ and $-n \delta_{1}<$ $\alpha<n \delta_{2}$, where $\delta_{1}, \delta_{2}$ are the constants in Lemma 2.5, then the operator $\mu_{\Omega, \lambda}^{*}$ is bounded from $\dot{K}_{p_{1}(\cdot)}^{\alpha, q_{2}}\left(w^{p_{1}(\cdot)}\right)$ to $\dot{K}_{p_{1}(\cdot)}^{\alpha, q_{1}}\left(w^{p_{1}(\cdot)}\right)$.

Remark 3.1. As it is well known that, $\mu_{\Omega, s}^{\rho} f(x) \leq 2^{n \lambda} \mu_{\Omega, \lambda}^{*, \rho} f(x)$ (see [6], p.89). Therefore, we give only the proof of Theorem 3.2.

\section{Proof of Theorem 3.2}

Let $f \in \dot{K}_{p_{1}(\cdot)}^{\alpha, q_{1}}\left(w^{p_{1}(\cdot)}\right)$. By the Jensen inequality, we have

$$
\begin{aligned}
\left\|\mu_{\Omega, \lambda}^{*, \rho} f\right\|_{\dot{K}_{p_{1}(\cdot)}^{\alpha, q_{2}}\left(w^{\left.p_{1}(\cdot)\right)}\right.}^{q_{1}} & \leq\left(\sum_{k=-\infty}^{\infty} 2^{\alpha q_{2} k}\left\|\left(\mu_{\Omega, \lambda}^{*, \rho} f\right) \chi_{k}\right\|_{L^{p_{1}(\cdot)}\left(w^{p_{1}(\cdot)}\right)}^{q_{2}}\right)^{\frac{q_{1}}{q_{2}}} \\
& \leq \sum_{k=-\infty}^{\infty} 2^{\alpha q_{1} k}\left\|\left(\mu_{\Omega, \lambda}^{*, \rho} f\right) \chi_{k}\right\|_{L^{p_{1}(\cdot)}\left(w^{p_{1}(\cdot)}\right)}^{q_{1}} .
\end{aligned}
$$

Denote $f_{j}=f \chi_{j}$ for each $j \in z$, then $f=\sum_{j=-\infty}^{\infty} f_{j}$, so we have

$$
\begin{aligned}
& \left\|\mu_{\Omega, \lambda}^{*, \rho} f\right\|_{\dot{K}_{p_{1}(\cdot)}^{\alpha, q_{2}}\left(w^{p_{1}(\cdot)}\right)}^{q_{1}} \leq \sum_{k=-\infty}^{\infty} 2^{\alpha q_{1} k}\left\|\left(\mu_{\Omega, \lambda}^{*, \rho} f\right) \chi_{k}\right\|_{L^{p_{1}(\cdot)}\left(w^{p_{1}(\cdot)}\right)}^{q_{1}} \\
& \leq \sum_{k=-\infty}^{\infty} 2^{\alpha q_{1} k}\left(\sum_{j=-\infty}^{k-2}\left\|\left(\mu_{\Omega, \lambda}^{*, \rho} f_{j}\right) \chi_{k}\right\|_{L^{p_{1}(\cdot)}\left(w^{p_{1}(\cdot)}\right)}\right)^{q_{1}} \\
& \leq \sum_{k=-\infty}^{\infty} 2^{\alpha q_{1} k}\left(\sum_{j=k-2}^{k+2}\left\|\left(\mu_{\Omega, \lambda}^{*, \rho} f_{j}\right) \chi_{k}\right\|_{L^{p_{1}(\cdot)}\left(w^{p_{1}(\cdot)}\right)}\right)^{q_{1}} \\
& \leq \sum_{k=-\infty}^{\infty} 2^{\alpha q_{1} k}\left(\sum_{j=k+2}^{\infty}\left\|\left(\mu_{\Omega, \lambda}^{*, \rho} f_{j}\right) \chi_{k}\right\|_{L^{p_{1}(\cdot)}\left(w^{p_{1}(\cdot)}\right)}\right)^{q_{1}} \\
& =: \mathrm{L}_{1}+\mathrm{L}_{2}+\mathrm{L}_{3} \text {. }
\end{aligned}
$$

First, we consider $\mathrm{L}_{2}$. Using Lemma 2.1 and $-2 \leq k-j \leq 2$, it is easy to get

$$
\begin{aligned}
\mathrm{L}_{2} & =\sum_{k=-\infty}^{\infty} 2^{\alpha q_{1} k}\left(\sum_{j=k-2}^{k+2}\left\|\left(\mu_{\Omega, \lambda}^{*, \rho} f_{j}\right) \chi_{k}\right\|_{L^{p_{1}(\cdot)}\left(w^{p_{1}(\cdot)}\right)}\right)^{q_{1}} \\
& =\sum_{k=-\infty}^{\infty}\left(\sum_{j=k-2}^{k+2} 2^{\alpha(k-j)} 2^{\alpha j}\left\|f_{j}\right\|_{L^{p_{1}(\cdot)}\left(w^{p_{1}(\cdot)}\right)}\right)^{q_{1}}
\end{aligned}
$$




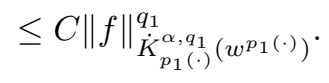

Now we need to consider $\mu_{\Omega, \lambda}^{*, \rho} f_{j}$. Applying the Minkowski inequality, we conclude that

$$
\begin{aligned}
& \left|\mu_{\Omega, \lambda}^{*, \rho}\left(f_{j}\right)(x)\right|= \\
& \left(\int_{0}^{\infty} \int_{\mathbb{R}^{n}}\left(\frac{t}{t+|x-y|}\right)^{\lambda n}\left|\frac{1}{t^{\rho}} \int_{|y-z| \leq t} \frac{\Omega(y, y-z)}{|y-z|^{n-\rho}} f_{j}(z) \mathrm{d} z\right|^{2} \frac{\mathrm{d} y \mathrm{~d} t}{t^{n+1}}\right)^{\frac{1}{2}} \\
& \quad \leq \int_{\mathbb{R}^{n}} f_{j}(z)\left(\int_{0}^{\infty} \int_{|y-z| \leq t}\left(\frac{t}{t+|x-y|}\right)^{\lambda n} \frac{|\Omega(y, y-z)|^{2}}{|y-z|^{2 n-2 \rho}} \frac{\mathrm{d} y \mathrm{~d} t}{t^{2 \rho+n+1}}\right)^{\frac{1}{2}} \mathrm{~d} z \\
& \quad \leq \int_{\mathbb{R}^{n}} f_{j}(z)\left(\int_{0}^{|x-z|} \int_{|y-z| \leq t}\left(\frac{t}{t+|x-y|}\right)^{\lambda n} \frac{|\Omega(y, y-z)|^{2}}{|y-z|^{2 n-2 \rho}} \frac{\mathrm{d} y \mathrm{~d} t}{t^{2 \rho+n+1}}\right)^{\frac{1}{2}} \mathrm{~d} z \\
& \quad+\int_{\mathbb{R}^{n}} f_{j}(z)\left(\int_{|x-z|}^{\infty} \int_{|y-z| \leq t}\left(\frac{t}{t+|x-y|}\right)^{\lambda n} \frac{|\Omega(y, y-z)|^{2}}{|y-z|^{2 n-2 \rho}} \frac{\mathrm{d} y \mathrm{~d} t}{t^{2 \rho+n+1}}\right)^{\frac{1}{2}} \mathrm{~d} z .
\end{aligned}
$$

For $\Omega \in L^{\infty}\left(\mathbb{R}^{n}\right) \times L^{2}\left(S^{n-1}\right)$ and $2 \rho-n>0$, the following inequality holds

$$
\begin{aligned}
\int_{|y-z| \leq t} \frac{|\Omega(y, y-z)|^{2}}{|y-z|^{2 n-2 \rho}} \mathrm{d} y & \leq \int_{s^{n-1}} \int_{0}^{t} \frac{\left|\Omega\left(s y^{\prime}+z, y^{\prime}\right)\right|^{2}}{s^{2 n-2 \rho}} s^{n-1} \mathrm{~d} s \mathrm{~d} \sigma\left(y^{\prime}\right) \\
& \leq\|\Omega\|_{L^{\infty}\left(\mathbb{R}^{n}\right) \times L^{2}\left(S^{n-1}\right)}^{2} t^{2 \rho-n} .
\end{aligned}
$$

Since $|x-z| \leq|x-y|+|y-z| \leq|x-y|+t$. For $\lambda>2$, taking $0<\delta<(\lambda-2) n$, we have

$$
\begin{aligned}
& \int_{0}^{|x-z|} \int_{|y-z| \leq t}\left(\frac{t}{t+|x-y|}\right)^{\lambda n} \frac{|\Omega(y, y-z)|^{2}}{|y-z|^{2 n-2 \rho}} \frac{\mathrm{d} y \mathrm{~d} t}{t^{2 \rho+n+1}} \\
& \leq \int_{0}^{|x-z|} \int_{|y-z| \leq t}\left(\frac{t}{t+|x-y|}\right)^{\lambda n-2 n-\delta} \frac{1}{|x-z|^{2 n+\delta}} \\
& \times \frac{|\Omega(y, y-z)|^{2}}{|y-z|^{2 n-2 \rho}} \frac{\mathrm{d} y \mathrm{~d} t}{t^{2 \rho-n-\delta+1}} \\
& \leq \frac{1}{|x-z|^{2 n+\delta}} \int_{0}^{|x-z|} \int_{|y-z| \leq t} \frac{|\Omega(y, y-z)|^{2}}{|y-z|^{2 n-2 \rho}} \frac{\mathrm{d} y \mathrm{~d} t}{t^{2 \rho-n-\delta+1}} \\
& \leq \frac{\|\Omega\|_{L^{\infty}\left(\mathbb{R}^{n}\right) \times L^{2}\left(S^{n-1}\right)}^{|x-z|}}{|x-z|^{2 n+\delta}} \int_{0}^{\delta-1} \mathrm{~d} t \\
& \leq C|x-z|^{-2 n} .
\end{aligned}
$$

If we take $1<\lambda_{1}<2$, then $\lambda_{1} n-n>0$ and $\lambda_{1} n-2 n<0$, so we have

$$
\begin{aligned}
& \int_{|x-z|}^{\infty} \int_{|y-z| \leq t}\left(\frac{t}{t+|x-y|}\right)^{\lambda n} \frac{|\Omega(y, y-z)|^{2}}{|y-z|^{2 n-2 \rho}} \frac{\mathrm{d} y \mathrm{~d} t}{t^{2 \rho+n+1}} \\
& \leq \int_{|x-z|}^{\infty} \int_{|y-z| \leq t}|x-z|^{-\lambda_{1} n} \frac{|\Omega(y, y-z)|^{2}}{|y-z|^{2 n-2 \rho}} \frac{\mathrm{d} y \mathrm{~d} t}{t^{2 \rho-\lambda_{1} n+n+1}}
\end{aligned}
$$




$$
\begin{aligned}
& \leq \int_{|x-z|}^{\infty}|x-z|^{-\lambda_{1} n} \int_{|y-z| \leq t} \frac{|\Omega(y, y-z)|^{2}}{|y-z|^{2 n-\lambda_{1} n}} \frac{\mathrm{d} y \mathrm{~d} t}{t^{n+1}} \\
& \leq \int_{|x-z|}^{\infty}|x-z|^{-\lambda_{1} n} \int_{s^{n-1}} \int_{0}^{t} \frac{\left|\Omega\left(y^{\prime},(y-z)^{\prime}\right)\right|^{2}}{s^{2 n-\lambda_{1} n}} s^{n-1} \mathrm{~d} s \mathrm{~d} \sigma\left(y^{\prime}\right) \frac{\mathrm{d} t}{t^{n+1}} \\
& \leq C\|\Omega\|_{L^{\infty}\left(\mathbb{R}^{n}\right) \times L^{2}\left(S^{n-1}\right)}^{2}|x-z|^{-\lambda_{1} n} \int_{|x-z|}^{\infty} t^{\lambda_{1} n-2 n-1} \mathrm{~d} t \\
& \leq C|x-z|^{-2 n} .
\end{aligned}
$$

Combining the above two estimates, we obtain

$$
\mu_{\Omega, \lambda}^{*}(f)(x) \leq \int_{\mathbb{R}^{n}} \frac{|f(z)|}{|x-z|^{n}} \mathrm{~d} z .
$$

Next, we consider $\mathrm{L}_{1}$. Noting that for $x \in A_{k}, z \in A_{j}$ and $j \leq k-2$, then $|x-z| \sim|x|$. By the virtue of the generalized Hölder inequality, we have

$$
\mu_{\Omega, \lambda}^{*, \rho}\left(f_{j}\right)(x) \leq C 2^{-k n}\left\|f_{j}\right\|_{L^{p_{1}(\cdot)}\left(w^{p_{1}(\cdot)}\right)}\left\|\chi_{j}\right\|_{\left(L^{p_{1}(\cdot)}\left(w^{p_{1}(\cdot)}\right)\right)^{\prime}} \cdot
$$

Applying Lemma 2.3 and Lemma 2.5 , we take $\|\cdot\|_{L^{p_{1}(\cdot)}\left(w^{p_{1}(\cdot)}\right)}$ for each side, we have

$$
\begin{aligned}
& \left\|\mu_{\Omega, \lambda}^{*, \rho}\left(f_{j}\right)(x) \chi_{k}\right\|_{L^{p_{1}(\cdot)}\left(w^{p_{1}(\cdot)}\right)} \\
& \leq C 2^{-k n}\left\|f_{j}\right\|_{L^{p_{1}(\cdot)}\left(w^{p_{1}(\cdot)}\right)}\left\|\chi_{j}\right\|_{\left(L^{p_{1}(\cdot)}\left(w^{p_{1}(\cdot)}\right)\right)^{\prime}}\left\|\chi_{k}\right\|_{L^{p_{1}(\cdot)}\left(w^{p_{1}(\cdot)}\right)} \\
& \leq C 2^{-k n}\left\|f_{j}\right\|_{L^{p_{1}(\cdot)}\left(w^{\left.p_{1}(\cdot)\right)}\right)}\left\|\chi_{B_{j}}\right\|_{\left(L ^ { p _ { 1 } ( \cdot ) } \left(w^{\left.\left.p_{1}(\cdot)\right)\right)^{\prime}}\right.\right.}\left\|\chi_{B_{k}}\right\|_{L^{p_{1}(\cdot)}\left(w^{\left.p_{1}(\cdot)\right)}\right)} \\
& \leq C\left\|f_{j}\right\|_{L^{p_{1}(\cdot)}\left(w^{\left.p_{1}(\cdot)\right)}\right)}\left\|\chi_{B_{j}}\right\|_{\left(L^{p_{1}(\cdot)}\left(w^{p_{1}(\cdot)}\right)\right)^{\prime}}\left\|\chi_{B_{k}}\right\|_{\left(L^{p_{1}(\cdot)}\left(w^{p_{1}(\cdot)}\right)\right)^{\prime}}^{-1} \\
& \leq C\left\|f_{j}\right\|_{L^{p_{1}(\cdot)}\left(w^{p_{1}(\cdot)}\right)}\left\|\chi_{B_{j}}\right\|_{\left(L^{p_{1}(\cdot)}\left(w^{p_{1}(\cdot)}\right)\right)^{\prime}}\left\|\chi_{B_{k}}\right\|_{\left(L^{p_{1}(\cdot)}\left(w^{p_{1}(\cdot)}\right)\right)^{\prime}} \\
& \leq C 2^{(j-k) n \delta_{2}}\left\|f_{j}\right\|_{L^{p_{1}(\cdot)}\left(w^{p_{1}(\cdot)}\right)} .
\end{aligned}
$$

Thus, we have

$$
\begin{aligned}
\mathrm{L}_{1} & \leq C \sum_{k=-\infty}^{\infty} 2^{\alpha q_{1} k}\left(\sum_{j=-\infty}^{k-2} 2^{(j-k) n \delta_{2}}\left\|f_{j}\right\|_{L^{p_{1}(\cdot)}\left(w^{p_{1}(\cdot)}\right)}\right)^{q_{1}} \\
& \leq C \sum_{k=-\infty}^{\infty}\left(\sum_{j=-\infty}^{k-2} 2^{j \alpha} 2^{(k-j)\left(\alpha-n \delta_{2}\right)}\left\|f_{j}\right\|_{L^{p_{1}(\cdot)}\left(w^{p_{1}(\cdot)}\right)}\right)^{q_{1}} .
\end{aligned}
$$

Now we have two cases: $1<q_{1}<\infty$ and $0<q_{1} \leq 1$. When $1<q_{1}<\infty$, by using the Hölder inequality, we have

$$
\mathrm{L}_{1} \leq C \sum_{k=-\infty}^{\infty}\left(\sum_{j=-\infty}^{k-2} 2^{j \alpha} 2^{(k-j)\left(\alpha-n \delta_{2}\right)}\left\|f_{j}\right\|_{L^{p_{1}(\cdot)}\left(w^{\left.p_{1}(\cdot)\right)}\right)}\right)^{q_{1}}
$$




$$
\begin{aligned}
& \leq C \sum_{k=-\infty}^{\infty}\left(\sum_{j=-\infty}^{k-2} 2^{j \alpha q_{1}} 2^{(k-j)\left(\alpha-n \delta_{2}\right) q_{1} / 2}\left\|f_{j}\right\|_{L^{p_{1}(\cdot)}\left(w^{p_{1}(\cdot)}\right)}^{q_{1}}\right) \\
& \times\left(\sum_{j=-\infty}^{k-2} 2^{(k-j)\left(\alpha-n \delta_{2}\right) q_{1}^{\prime} / 2}\right)^{q_{1} / q_{1}^{\prime}} \\
& \leq C \sum_{k=-\infty}^{\infty} \sum_{j=-\infty}^{k-2} 2^{j \alpha q_{1}} 2^{(k-j)\left(\alpha-n \delta_{2}\right) q_{1} / 2}\left\|f_{j}\right\|_{L^{p_{1}(\cdot)}\left(w^{p_{1}(\cdot)}\right)}^{q_{1}} \\
& \leq C \sum_{j=-\infty}^{\infty} 2^{j \alpha q_{1}}\left\|f_{j}\right\|_{L^{p_{1}(\cdot)}\left(w^{p_{1}(\cdot)}\right)}^{q_{1}} \sum_{k=j+2}^{\infty} 2^{(k-j)\left(\alpha-n \delta_{2}\right) q_{1} / 2} \\
& \leq C\|f\|_{K_{p_{1}(\cdot)}^{\alpha, q_{1}}\left(w^{p_{1}(\cdot)}\right)^{\cdot}}^{q_{1}}
\end{aligned}
$$

When $0<q_{1} \leq 1$, again by the Jensen inequality, we obtain

$$
\begin{aligned}
& \mathrm{L}_{1} \leq C \sum_{k=-\infty}^{\infty}\left(\sum_{j=-\infty}^{k-2} 2^{j \alpha} 2^{(k-j)\left(\alpha-n \delta_{2}\right)}\left\|f_{j}\right\|_{L^{p_{1}(\cdot)}\left(w^{p_{1}(\cdot)}\right)}\right)^{q_{1}} \\
& \leq C \sum_{k=-\infty}^{\infty} \sum_{j=-\infty}^{k-2} 2^{j \alpha q_{1}} 2^{(k-j)\left(\alpha-n \delta_{2}\right) q_{1}}\left\|f_{j}\right\|_{L^{p_{1}(\cdot)}\left(w^{p_{1}(\cdot)}\right)}^{q_{1}} \\
& \leq C \sum_{j=-\infty}^{\infty} 2^{j \alpha q_{1}}\left\|f_{j}\right\|_{L^{p_{1}(\cdot)}\left(w^{p_{1}(\cdot)}\right)}^{q_{1}} \sum_{k=j+2}^{\infty} 2^{(k-j)\left(\alpha-n \delta_{2}\right) q_{1}} \\
& \leq C\|f\|_{\dot{K}_{p_{1}(\cdot)}^{\alpha, q_{1}}\left(w^{p_{1}(\cdot)}\right)}^{q_{1}} .
\end{aligned}
$$

Finally, we estimate $\mathrm{L}_{3}$. Noting that for $x \in A_{k}, y \in A_{j}$ and $j \geq k+2$, then $|y-x| \sim|y|$. By the virtue of the generalized Hölder inequality, we have

$$
\mu_{\Omega, \lambda}^{*, \rho}\left(f_{j}\right)(x) \leq C 2^{-j n}\left\|f_{j}\right\|_{L^{p_{1}(\cdot)}\left(w^{p_{1}(\cdot)}\right)}\left\|\chi_{j}\right\|_{\left(L^{p_{1}(\cdot)}\left(w^{p_{1}(\cdot)}\right)\right)^{\prime}}
$$

Applying Lemma 2.3 and Lemma 2.5 , we can take $\|\cdot\|_{L^{p_{1}(\cdot)}\left(w^{p_{1}(\cdot)}\right)}$ for each side, we have

$$
\begin{aligned}
& \left\|\mu_{\Omega, \lambda}^{*, \rho}\left(f_{j}\right)(x) \chi_{k}\right\|_{L^{p_{1}(\cdot)}\left(w^{p_{1}(\cdot)}\right)} \\
& \leq C 2^{-j n}\left\|f_{j}\right\|_{L^{p_{1}(\cdot)}\left(w^{p_{1}(\cdot)}\right)}\left\|\chi_{j}\right\|_{\left(L^{p_{1}(\cdot)}\left(w^{\left.p_{1}(\cdot)\right)}\right)\right.}\left\|\chi_{k}\right\|_{L^{p_{1}(\cdot)}\left(w^{p_{1}(\cdot)}\right)} \\
& \leq C 2^{-j n}\left\|f_{j}\right\|_{L^{p_{1}(\cdot)}\left(w^{\left.p_{1}(\cdot)\right)}\right)}\left\|\chi_{B_{j}}\right\|_{\left(L ^ { p _ { 1 } ( \cdot ) } \left(w^{\left.\left.p_{1}(\cdot)\right)\right)^{\prime}}\right.\right.}\left\|\chi_{B_{k}}\right\|_{L^{p_{1}(\cdot)}\left(w^{p_{1}(\cdot)}\right)} \\
& \leq C\left\|f_{j}\right\|_{L^{p_{1}(\cdot)}\left(w^{p_{1}(\cdot)}\right)}\left\|\chi_{B_{k}}\right\|_{\left(L^{p_{1}(\cdot)}\left(w^{p_{1}(\cdot)}\right)\right)}\left\|\chi_{B_{j}}\right\|_{\left(L^{p_{1}(\cdot)}\left(w^{p_{1}(\cdot)}\right)\right)}^{-1} \\
& \leq C\left\|f_{j}\right\|_{L^{p_{1}(\cdot)}\left(w^{p_{1}(\cdot)}\right)}\left\|\chi_{B_{k}}\right\|_{\left(L^{p_{1}(\cdot)}\left(w^{p_{1}(\cdot)}\right)\right)} \\
& \left\|\chi_{B_{j}}\right\|_{\left(L^{p_{1}(\cdot)}\left(w^{p_{1}(\cdot)}\right)\right)} \\
& \leq C 2^{(k-j) n \delta_{1}}\left\|f_{j}\right\|_{L^{p_{1}(\cdot)}\left(w^{p_{1}(\cdot)}\right)} .
\end{aligned}
$$


Thus, we have

$$
\begin{aligned}
\mathrm{L}_{3} & \leq C \sum_{k=-\infty}^{\infty} 2^{\alpha q_{1} k}\left(\sum_{j=k+2}^{\infty} 2^{(k-j) n \delta_{1}}\left\|f_{j}\right\|_{L^{p_{1}(\cdot)}\left(w^{p_{1}(\cdot)}\right)}\right)^{q_{1}} \\
& \leq C \sum_{k=-\infty}^{\infty}\left(\sum_{j=k+2}^{\infty} 2^{j \alpha} 2^{(k-j)\left(\alpha+n \delta_{1}\right)}\left\|f_{j}\right\|_{L^{p_{1}(\cdot)}\left(w^{p_{1}(\cdot)}\right)}\right)^{q_{1}} .
\end{aligned}
$$

Now we also have two cases: $1<q_{1}<\infty$ and $0<q_{1} \leq 1$. When $1<q_{1}<\infty$, by using the Hölder inequality, we have

$$
\begin{aligned}
\mathrm{L}_{3} & \leq C \sum_{k=-\infty}^{\infty}\left(\sum_{j=k+2}^{\infty} 2^{j \alpha} 2^{(k-j)\left(\alpha+n \delta_{1}\right)}\left\|f_{j}\right\|_{L^{p_{1}(\cdot)}\left(w^{p_{1}(\cdot)}\right)}\right)^{q_{1}} \\
& \leq C \sum_{k=-\infty}^{\infty}\left(\sum_{j=k+2}^{\infty} 2^{j \alpha q_{1}} 2^{(k-j)\left(\alpha+n \delta_{1}\right) q_{1} / 2}\left\|f_{j}\right\|_{L^{p_{1}(\cdot)}\left(w^{\left.p_{1}(\cdot)\right)}\right)}^{q_{1}}\right) \\
& \times\left(\sum_{j=k+2}^{\infty} 2^{(k-j)\left(\alpha+n \delta_{1}\right) q_{1}^{\prime} / 2}\right)^{q_{1} / q_{1}^{\prime}} \\
& \leq C \sum_{k=-\infty}^{\infty} \sum_{j=k+2}^{\infty} 2^{j \alpha q_{1}} 2^{(k-j)\left(\alpha+n \delta_{1}\right) q_{1} / 2}\left\|f_{j}\right\|_{L^{p_{1}(\cdot)}\left(w^{p_{1}(\cdot)}\right)}^{q_{1}} \\
& \leq C \sum_{j=-\infty}^{\infty} 2^{j \alpha q_{1}}\left\|f_{j}\right\|_{L^{p_{1}(\cdot)}\left(w^{\left.p_{1}(\cdot)\right)} \sum_{k=-\infty}^{q_{1}} 2^{(k-j)\left(\alpha+n \delta_{1}\right) q_{1} / 2}\right.} \\
& \leq C\|f\|_{\dot{K}_{p_{1}(\cdot)}^{\alpha, q_{1}}\left(w^{p_{1}(\cdot)}\right)}^{q_{1}} \cdot
\end{aligned}
$$

When $0<q_{1} \leq 1$, applying the Jensen inequality, we obtain

$$
\begin{aligned}
& \mathrm{L}_{3} \leq C \sum_{k=-\infty}^{\infty}\left(\sum_{j=k+2}^{\infty} 2^{j \alpha} 2^{(k-j)\left(\alpha+n \delta_{1}\right)}\left\|f_{j}\right\|_{L^{p_{1}(\cdot)}\left(w^{\left.p_{1}(\cdot)\right)}\right.}\right)^{q_{1}} \\
& \leq C \sum_{k=-\infty}^{\infty} \sum_{j=k+2}^{\infty} 2^{j \alpha q_{1}} 2^{(k-j)\left(\alpha+n \delta_{1}\right) q_{1}}\left\|f_{j}\right\|_{L^{p_{1}(\cdot)}\left(w^{p_{1}(\cdot)}\right)}^{q_{1}} \\
& \leq C \sum_{j=-\infty}^{\infty} 2^{j \alpha q_{1}}\left\|f_{j}\right\|_{L^{p_{1}(\cdot)}\left(w^{p_{1}(\cdot)}\right)}^{q_{1}} \sum_{k=-\infty}^{j-2} 2^{(k-j)\left(\alpha-n \delta_{2}\right) q_{1}} \\
& \leq C\|f\|_{K_{p_{1}(\cdot)}^{\alpha, q_{1}}\left(w^{p_{1}(\cdot)}\right)}^{q_{1}} .
\end{aligned}
$$

This completes the proof of Theorem 3.2. 


\section{Acknowledgments}

The authors are very grateful to the referees for their valuable comments.

\section{REFERENCES}

1. Kenig, C. E. (1994). Harmonic analysis techniques for second order elliptic boundary value problems (Vol. 83). American Mathematical Soc.

2. Cruz-Uribe, D. V., \& Fiorenza, A. (2013). Variable Lebesgue spaces: foundations and harmonic analysis. Springer Science \& Business Media.

3. Bennett, C., \& Sharpley, R. C. (1988). Interpolation of operators (Vol. 129). Academic press.

4. Shanzhen, L., Yong, D., \& Dunyan, Y. (2007). Singular integrals and related topics. World Scientific.

5. García-Cuerva, J., \& De Francia, J. R. (2011). Weighted norm inequalities and related topics (Vol. 116). Elsevier.

6. Stein, E. M. (2016). Singular integrals and differentiability properties of functions (PMS30) (Vol. 30). Princeton university press.

7. Ding, Y., Lin, C. C., \& Shao, S. (2004). On the Marcinkiewicz integral with variable kernels. Indiana University Mathematics Journal, 805-821.

8. Chen, J., Ding, Y., \& Fan, D. (2006). Littlewood-Paley operators with variable kernels. Science in China Series A, 49(5), 639-650.

9. Xue, Q., \& Ding, Y. (2009). Weighted estimates for the multilinear commutators of the Littlewood-Paley operators. Science in China Series A: Mathematics, 52(9), 1849-1868.

10. Hörmander, L. (1960). Estimates for translation invariant operators in $L^{p}$ spaces. Acta Mathematica, 104(1-2), 93-140.

11. Wei, X., \& Tao, S. (2013). Boundedness for parametrized Littlewood-Paley operators with rough kernels on weighted weak Hardy spaces. In Abstract and Applied Analysis (Vol. 2013). Hindawi.

12. Lin, Y., \& Xuan, X. (2016). Weighted boundedness for commutators of parameterized Littlewood-Paley operators and area integral. Publications de l'Institut Mathematique, 100(114)

13. Kováčik, O., \& Rákosník, J. (1991). On spaces $L^{p(x)}$ and $W^{k, p(x)}$. Czechoslovak Mathematical Journal, 41(4), 592-618.

14. Izuki, M. (2010). Boundedness of commutators on Herz spaces with variable exponent. Rendiconti del Circolo Matematico di Palermo, 59(2), 199-213.

15. Abdalmonem, A., Abdalrhman, O., \& Tao, S. (2016). Boundedness of fractional integral with variable kernel and their commutators on variable exponent Herz spaces. Applied Mathematics, 7, 1165-1182.

16. Wang, L., \& Tao, S. (2016). Parameterized Littlewood-Paley operators and their commutators on Herz spaces with variable exponent. Turkish Journal of Mathematics, 40(1), $122-145$.

17. Izuki, M. (2009). Herz and amalgam spaces with variable exponent, the Haar wavelets and greediness of the wavelet system. East journal on approximations, 15(1), 87-110.

18. ZHANG, P., \& WU, J. L. (2013). Boundedness of fractional Hardy type operators on HerzMorrey spaces with variable exponent. Math. Pract. Theory, 43, 247-254.

19. Samko, S.: Variable exponent Herz spaces. Mediterr. J. Math., 10(4), 2007C2025(2013).

20. Diening, L.: Maximal functions on generalized Lebesgue spaces $L^{p(\cdot)}$. Math. Inequal. Appl. 7(2), 245C253(2004)

21. Diening, L., \& Hästö, P. (2008). Muckenhoupt weights in variable exponent spaces. preprint. 
22. D Cruz-Uribe, S. F. O., Fiorenza, A., \& Neugebauer, C. J. (2012). Weighted norm inequalities for the maximal operator on variable Lebesgue spaces. Journal of Mathematical Analysis and Applications, 394(2), 744-760.

23. Cruz-Uribe, D., Diening, L., \& Hästö, P. (2011). The maximal operator on weighted variable Lebesgue spaces. Fractional Calculus and Applied Analysis, 14(3), 361-374.

24. Izuki, M. (2013). Remarks on Muckenhoupt weights with variable exponent. Journal of Analysis and Applications, 11(1), 27-41.

25. Cruz-Uribe, D., \& Wang, L. A. (2017). Extrapolation and weighted norm inequalities in the variable Lebesgue spaces. Transactions of the American Mathematical Society, 369(2), 1205-1235.

26. Izuki, M., \& Noi, T. (2016). Boundedness of fractional integrals on weighted Herz spaces with variable exponent. Journal of Inequalities and Applications, 2016(1), 199.

27. Diening, L. (2004). Maximal function on generalized Lebesgue spaces $L^{p(\cdot)}$. Math. Ineq. App., 7, 245-253.

28. Cruz-Uribe, D., Fiorenza, A., \& Neugebauer, C. J. (2003). The maximal function on variable $L^{p}$ spaces. Ann. Acad. Sci. Fenn. Math., 28(1), 223-238.

29. Karlovich, A. Y., \& Spitkovsky, I. M. (2014). The Cauchy singular integral operator on weighted variable Lebesgue spaces. In Concrete Operators, Spectral Theory, Operators in Harmonic Analysis and Approximation (pp. 275-291). Birkhuser, Basel.

30. Muckenhoupt, B. (1972). Weighted norm inequalities for the Hardy maximal function. Transactions of the American Mathematical Society, 207-226.

31. Lu, S., Yang, D., \& Hu, G. (2008). Herz type spaces and their applications. Science Press.

32. Komori, Y., \& Matsuoka, K. (2009). Boundedness of several operators on weighted Herz spaces. Journal of Function Spaces, 7(1), 1-12.

33. Izuki, M., \& Noi, T. (2016). An intrinsic square function on weighted Herz spaces with variable exponent. Journal of Mathematical Inequalities, 11, 49-58.

34. Kozo, Y. (1996). Boundedness of Littlewood-Paley operators. Mathematica japonicae, 43(1), 143-150.

\section{Afif Abdalmonem}

Faculty of Science, University of Dalanj, Dalanj, Sudan.

e-mail: afeefy86@gmail.com

Omer Abdalrhman

College of Education, Shendi University, Shendi, Sudan.

e-mail: humoora@gmail.com

Shuangping Tao

Northwest Normal University, Lanzhou, China.

e-mail: taosp@nwnu.edu.cn 